\title{
Robust correlation tracker
}

\section{SANKAR KISHORE and K VEERABHADRA RAO}

\author{
Research Centre Imarat, Vignyanakancha PO, Hyderabad 500 069, India
}

MS received 13 December 1999

\begin{abstract}
Correlation tracking plays an important role in the automation of weapon systems. Area correlation is an effective technique for tracking targets that have neither prominent features nor high contrast with the background and the 'target' can even be an area or a scene of interest. Even though this technique is robust under varying conditions of target background and light conditions, it has some problems like target drift and false registration. When the tracker or target is moving, the registration point drifts due to the discrete pixel size and aspect angle change.

In this research work, an attempt has been made to improve the performance of a correlation tracker for tracking ground targets with very poor contrast. In the present work only the CCD visible images with very poor target to background contrast are considered.

Applying novel linear and nonlinear filters, the problems present in the correlation tracker are overcome. Confidence and redundancy measures have been proposed to improve the performance by detecting misregistration. The proposed algorithm is tested on different sequences of images and its performance is satisfactory.
\end{abstract}

Keywords. Image processing; correlation; trackers.

\section{Introduction}

Correlation tracking plays an important role in the automation of a weapon system. During the last two decades, a lot of research work is going on in the developing and implementation of algorithms. Area Correlation Algorithm (ACA) is one of the popular techniques used in correlation trackers. ACA has been chosen for this application based on its performance against low contrast images, noise, scale changes etc (Kumar et al 1987).

ACA is an effective technique for tracking targets that have neither prominent features nor high contrast with the background and the target can be even an area or a scene of interest. Area correlation consists of overlapping a reference image over a search image and computing a similarity function. The window within the search image having 
maximum similarity with the reference is taken to be the target's location (Kumar et al 1987; Venkateswarlu et al 1992). A number of image registration algorithms and schemes are reported in the literature (Anuta 1970; Barnea \& Silverman 1972; Pratt 1974).

In the fields of reconnaissance, some practical problems such as change in target-image size, viewing angle, contrast variations, shape and texture of objects, occlusion of target etc., are encountered as the imaging sensor approaches or recedes from the target area. A periodic updating of the reference target-image circumvents these problems. Otherwise, the image-tracking algorithm may become divergent with time, thereby losing the track (Venkateswarlu et al 1992).

In this paper, different techniques, both with linear and nonlinear filters/masks, are proposed/developed to overcome problems like drift and false registration. Section 2 briefly describes the area correlation algorithm and the associated problems. The techniques developed are described in $\S 3$. To achieve reliable performance some kind of confidence measures are discussed in $\S 4$. The performance of the developed techniques on sequences of very low contrast images is presented in $\$ 5$.

\section{Area correlation algorithm (ACA)}

ACA is chosen for implementation because of its superior performance (Kumar et al 1987). The algorithm is as follows.

Let $S$ be the search image of $N \times N$ size and $R$ be the reference image of $M \times M$ size $(M<N)$.

Each pixel takes one of $G$ gray levels. i.e.,

$0 \leq R(l, m) \leq G-1, \quad$ for $1 \leq l, m \leq M$,

$0 \leq S(l, m) \leq G-1, \quad$ for $1 \leq l, m \leq N$.

The $M \times M$ window of the search image $S i j$ denotes starting at pixel $(i, j)$ i.e.,

$$
\begin{aligned}
\operatorname{Sij}(l, m)=S & (i+1-1, j+m-1), \\
& \quad \text { for } 1 \leq l, m \leq M, \\
& \text { for } 1 \leq l, m \leq N-M+1 .
\end{aligned}
$$

The normalized correlation function $C i j$ is computed between $R$ and $S i j$ by

$$
\begin{aligned}
& C i j=\sum_{l=0}^{M-1 M-1} \sum_{m=0}^{M} R(l, m) \operatorname{Sij}(l, m) /\left[\sum_{l=0}^{M-1} \sum_{m=0}^{M-1} R^{2}(l, m) \sum_{l=0}^{M-1} \sum_{m=0}^{M-1} S_{i j}^{2}(l, m)\right]^{1 / 2}, \\
& 0 \leq i, j \leq N-M .
\end{aligned}
$$

The maximum of $C i j$ gives the point of registration. $C i j$ lies between 0 and 1 (for a perfect match). The normalization is required, as the unnormalized correlation function dynamic range is very large. In such a situation, the unnormalized function can give a false peak even when the actual reference is present in the search image without any distortion or noise (Barnea \& Silverman 1972). 
As the distance between the imaging sensor and the target scene changes, the target area size and aspect angle change gradually. The reference area designated from the initial distance and look-angle completely differs as the image sensor approaches or recedes from the target area. Hence the reference is updated with the new image reference for the next frame and the old reference is discarded (Venkateswarlu et al 1992).

Even though ACA is robust under varying conditions of target, background and light conditions, this method needs augmentation of some special algorithms to overcome problems like target drift and false registration.

\subsection{False registration}

The point of highest correlation value is declared as the match point. In a frame, even if the target goes out of the field of view (FOV), some other area is declared as the match point and the reference is updated. In subsequent frames, even if the target comes back into the FOV, the correlation tracker does not go back to the original area. Thus the target can be missed due to any temporary disturbance in the image.

\subsection{Target drift}

The reference image contains background in addition to the target. Due to the discrete pixel size and due to target motion, the target may drift out of the reference image. This drift has to be corrected locally.

\section{An efficient edge detection based tracking algorithm}

In this work, an attempt has been made to improve the performance of the correlation tracker for tracking ground targets with very poor contrast. In this study, only visible images with very poor target-to-background contrast are considered.

The scheme basically consists of two parts. The first part consists of the application of the preprocessing techniques that facilitates the area correlation. The preprocessing consists of the following three steps in sequence.

- Stray/isolated pixel removal

- Edge detection

- Stray line removal.

The next step is to go for ACA followed by target centreing.

\subsection{Stray pixel removal}

Generally, tracker images may have very few uncorrelated noise pixels due to the electronics or EMI emanating from other electronics hardware. The elimination of these stray points is necessary, otherwise, during subsequent processing, single isolated points become big blobs as big as the target. These points may create obstructions in tracking the original target.

The most common way to look for discontinuities is to run a mask of $3 \times 3$ or $5 \times 5$ through the image. This procedure involves computing the sum of products of the coefficients with the gray levels contained in the region encompassed by the mask. It 
measures the weighted differences between the centre point and its neighbours. The idea is that the gray level of an isolated point will be quite different from the gray level of its neighbours. The emphasis here is strictly on the detection of points. That is, only that pixel whose difference from its neighbours is greater than some defined threshold $(T)$ is considered an isolated point. For this purpose various filters available in the literature (Phillips 1995; Gonzalez et al 1999) are not effective.

As a result, a method is developed for averaging in-site. The whole image is scanned to search for stray points. A stray point is located if the absolute difference from all its neighbours exceeds a threshold $(T)$. The threshold is kept at 60 for the present sequence. The figure 60 is arrived at after testing the algorithm on many image sequences. If such a point is found, its gray value is replaced by the average of its neighbours. The algorithm is as follows.

For $i=1$ to (height -1$)$

$$
\begin{aligned}
& \text { For } j=1 \text { to }(\text { width }-1) \\
& \text { If abs }((\text { image }[i][j]-\text { image }[i][j+1]) \& \& \\
& \quad \text { (image }[i][j]-\text { image }[i+1][j+1]) \& \& \\
& \quad \text { (image }[i][j]-\text { image }[i+1][j]>60)) \\
& \text { gray }=(\text { image }[i][j+1]+\text { image }[i+1][j+1]+\text { image }[i+1][j]) / 3 \\
& \text { else } \quad \\
& \text { gray }=\text { image }[i][j] .
\end{aligned}
$$

By this algorithm, the isolated/stray points are removed from the frames and passed to edge detection. The implemented edge technique is described below.

\subsection{Edge detection}

Edge detection by gradient operations tends to work well in cases involving images with sharp intensity transitions and relatively low noise. Zero crossing offers an alternative in cases when edges are blurry or when a high noise content is present.

Sobel operators have the advantage of providing both a differencing and a smoothing effect. Because derivatives enhance noise, the smoothing effect is a particularly attractive feature of Sobel operators. The Laplacian operator is unacceptably sensitive to noise. Moreover, the Laplacian operator produces double edges and is unable to detect edge direction.

The actual image received may not have any clear features. But on the other hand, the tracking algorithm would work better on images having distinct features. Edge detection using the Sobel operator has not given better performance as it considers only two directions, viz. $x$ and $y$. To detect the edges of the targets in all six directions, six masks, derived by rotating the Soble's operators, were used and gradient calculation was used to detect the edges. The suggested masks for the six directions are

$$
\begin{array}{rrrrrrrrrrrrrrrrrr}
1 & 2 & 1 & 1 & 0 & -1 & 2 & 1 & 0 & 0 & 1 & 2 & -2 & -1 & 0 & 0 & -1 & -2 \\
0 & 0 & 0 & 2 & 0 & -2 & 1 & 0 & -1 & -1 & 0 & 1 & -1 & 0 & 1 & 1 & 0 & -1 \\
-1 & -2 & -1 & 1 & 0 & -1 & 0 & -1 & -2 & -2 & -1 & 0 & 0 & 1 & 2 & 2 & 1 & 0
\end{array}
$$


Computation of the gradient at the location of the centre of the masks gives one value of the gradient. All the six masks are run through an image for the same point in the image and the maximum value of all the masks is taken in generating the gradient image. To get the next value, the masks are moved to the next pixel location and the procedure is repeated. Thus, after the procedure has been completed for all possible locations, the result is a gradient image of the same size as the original image. As usual, mask operations on the border of an image are implemented by using appropriate partial neighbourhoods.

In the original frames the vertical/horizontal bands have become one/two pixel wide vertical/horizontal lines respectively. The algorithm to remove these lines is described below.

\subsection{Stray line removal}

Due to the high shock and/or vibration some of the image frames may have black bands. By applying the edge techniques described in the earlier section, these bands become lines of one/two pixel thick horizontal/vertical lines. These lines are referred to as stray lines. In some of the cases, the received images may have straight lines passing through the frames. Such anomalies can cause a disturbance in the tracking algorithm.

In general, all the masks available in the literature (Phillips 1995; Gonzalez et al 1999) for highlighting the lines are moved all over the image to every possible positions. By using these masks, the horizontal/vertical lines present in the edged images become blurred, but cannot be removed. The present requirement is to remove the lines from the edged images.

This algorithm searches the image for horizontal straight lines and, if the absolute difference from its neighbouring pixels exceeds the threshold, it is replaced by the average of the total length of the neighbouring pixels. The same procedure is followed for the vertical lines also. The algorithm is as follows.

\section{For horizontal lines}

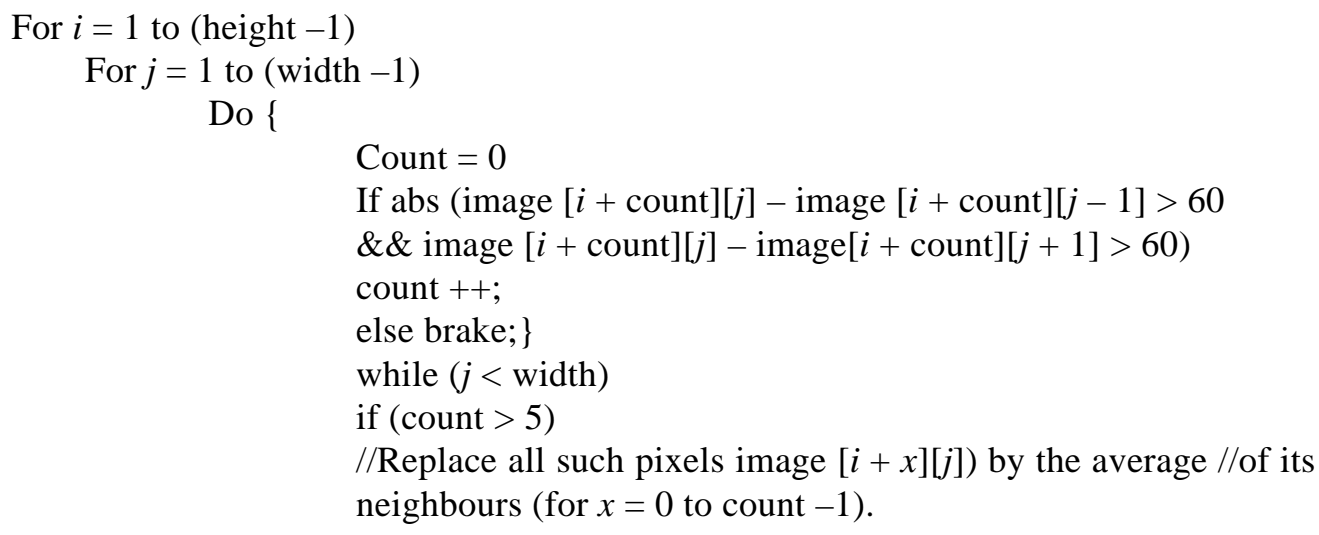


For vertical lines

$$
\begin{gathered}
\text { For } i=1 \text { to (height }-1) \\
\text { For } j=1 \text { to }(\text { width }-1) \\
\text { Do }\{
\end{gathered}
$$

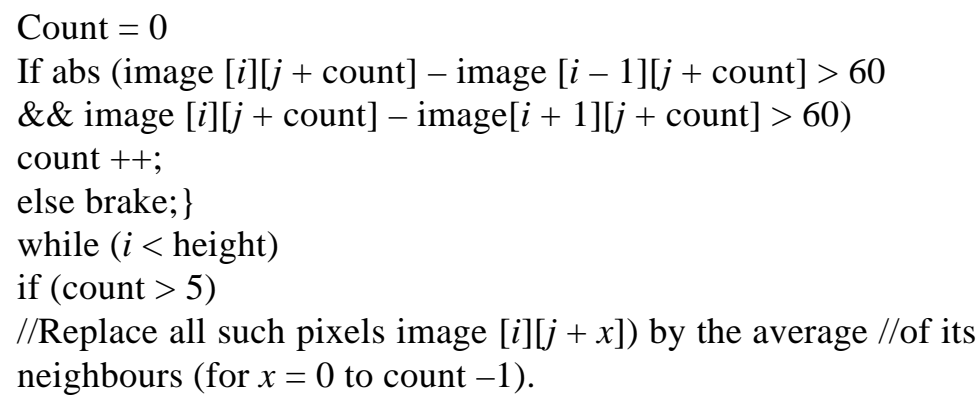

Once the image has been preprocessed, the correlation algorithm described in the earlier section may be applied, that is, on the edged image. To correct the drift present in the correlation tracker, the target-centreing technique is developed, which is described in the following section.

\subsection{Target centreing}

Due to discrete pixel size and due to target motion, the target drifts out of the reference image. This drift can be avoided by moving the target to the centre of every frame by using the target features.

The correlation algorithm gives the registration point as a best match. To obtain the threshold, consider a $32 \times 32$ window around the registration point (which is double the $16 \times 16$ reference frame) and calculate mean and maximum gray values. The threshold is taken as the average of mean and the maximum gray value. i.e.,

$$
\text { Threshold }=[\text { mean }+ \text { maximum gray value }] / 2 .
$$

Using the above computed threshold, the image is binarised and the edged picture is obtained. When we apply the above algorithm to real images, the binarized image contains only the target. To determine the location of the centre of the target, the target-image is fitted in a rectangle (parallel to either the $x$-axis or the $y$-axis) and the centre of the rectangle is taken as the centre of the target. The reference gate centre is accorded to the target centre. Thus the drift is eliminated. The image area (in which the target is at the centre) within the corrected gate is taken as reference image for the next frame.

\section{Confidence measures}

In order to achieve reliable performance, it is essential to have some kind of confidence measures built into the system. The correlation algorithm may fail to give correct registration because of low contrast of the reference, image noise, occlusion etc. In 
tracking applications, where the reference has to be updated frame by frame to reduce the effect of magnification, occlusion etc, even a single wrong registration will lead to false track. In such applications, the registration must function with some confidence. Confidence measures are some kind of criterion functions, which give the conformity of the registration. Researchers have proposed many confidence measures in the literature (Anandan 1985; Raju 1990).

In this present study, a new confidence measure is defined, to prevent false updation of the reference. By studying the variance and mean of different image sequences with and without target, the following confidence measure is proposed. The variance and mean of the current reference image are found. Since tracking is performed on the edged image, whenever the target is present in the reference gate only then the mean of the window is maximum, otherwise it is minimum. Similarly, variance is low if there is no target. The ratio between the old variance and new variance is considered as a confidence measure. Similarly, the ratio between old mean and new mean is considered another confidence measure. The reference image is updated only when their ratios are independently less than two. That is

$$
\text { Variance_old/Variance_new }<2 \& \& \text { Mean_old/mean_new }<2 \text {. }
$$

\section{Performance and results}

To evaluate the performance of the proposed algorithms/schemes, three available image sequences are used (figures 1-3). Each sequence contains nearly 80-90 frames. The size of the each frame is $100 \times 100$. Target size may be $2 \times 2$ app. Frame numbers are printed on each frame for reference. Each image frame has a 8-bit resolution and captured at a rate of $30 \mathrm{~ms}$.

It can be seen from the image frames that (a) frames have stray/isolated pixels, may be due to electronic noise (figures 1-3). (b) Frames can be shifted horizontally or vertically (figure 2) leaving a blank due to some obstruction as in the frames 4, 20, 24 etc. due to shock and vibration. (c) Frames can have one/two pixel wide horizontal/vertical lines (figure 2) as in the frames 20,33,53,54, 55 etc. may be due to loss of address/data synchronization.

The reference (target) image is selected from the first frame of the sequence and a white box is kept around it. This reference image is used in the next frames. The white boxes on the other frames indicate that the tracker has tracked the target and updated the reference, otherwise no update has taken place.

In some cases, the target is missing in the frame, and then the tracker has not updated the reference and used the earlier reference in the subsequent images. For example, in frame 5 (figure 2), the reference is used in frame 6 but is not updated. Similarly in frames 7 and 8, no reference is updated and in frame 9 the reference is updated.

In some cases, shift in the image frames has been taken care of by the algorithm and the tracker has not lost the target. The horizontal/vertical lines present in the frames have not affected tracking performance. The drift is also arrested and the target is always at the centre of the tracking gate/reference image. Thus the developed algorithm/scheme is robust in all respects. 


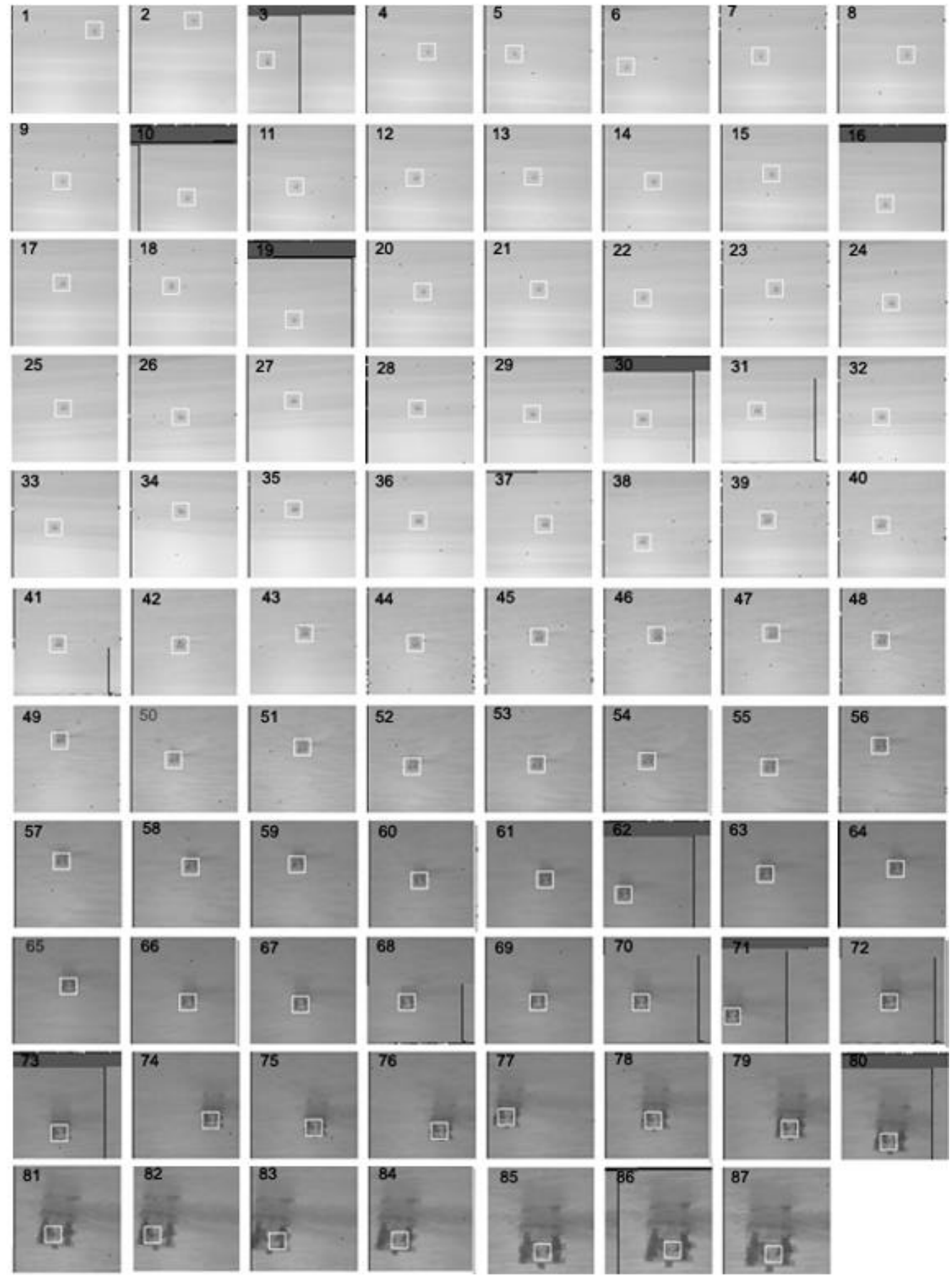

Figure 1. Image sequence I. 


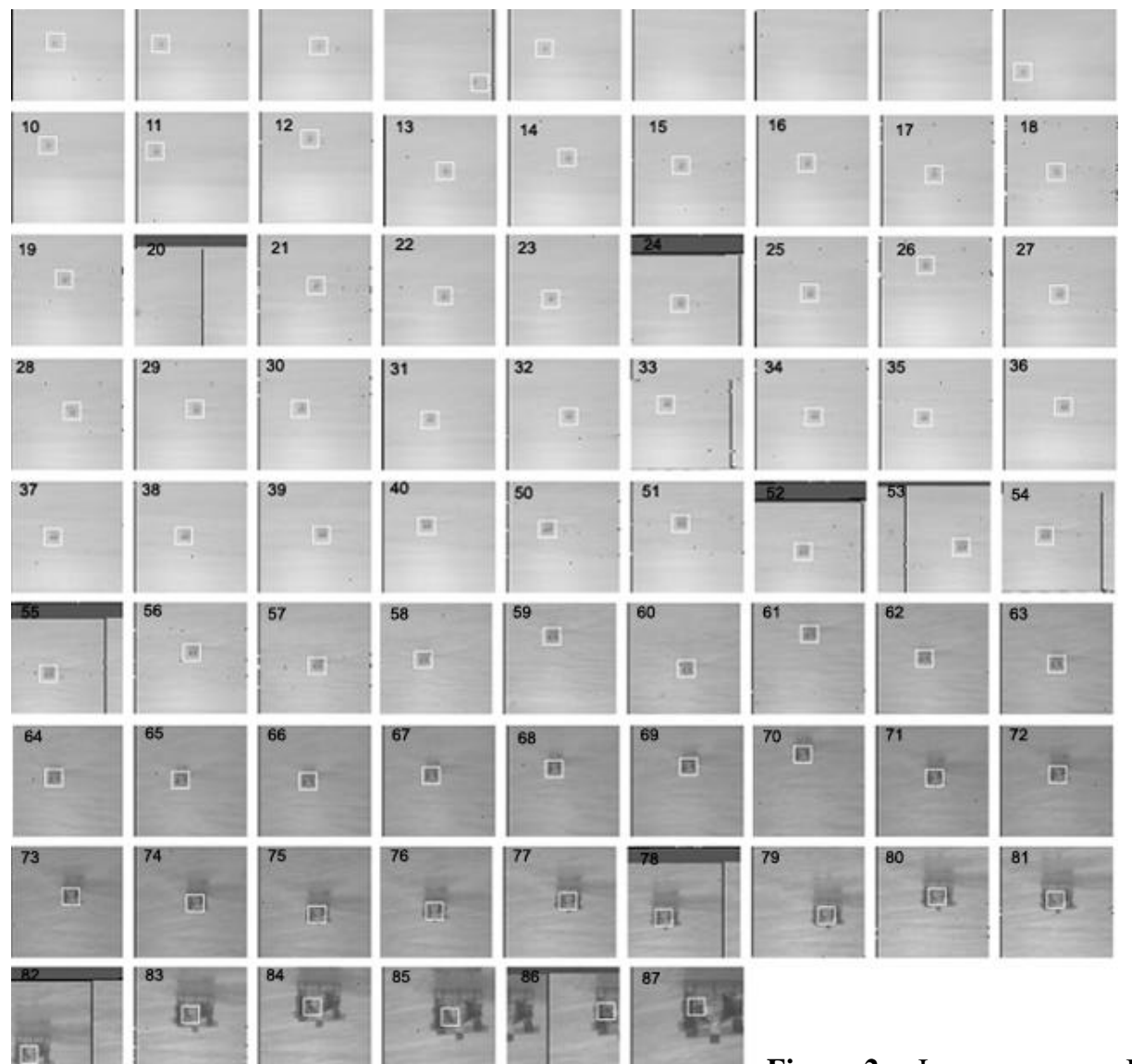

Figure 2. Image sequence II.
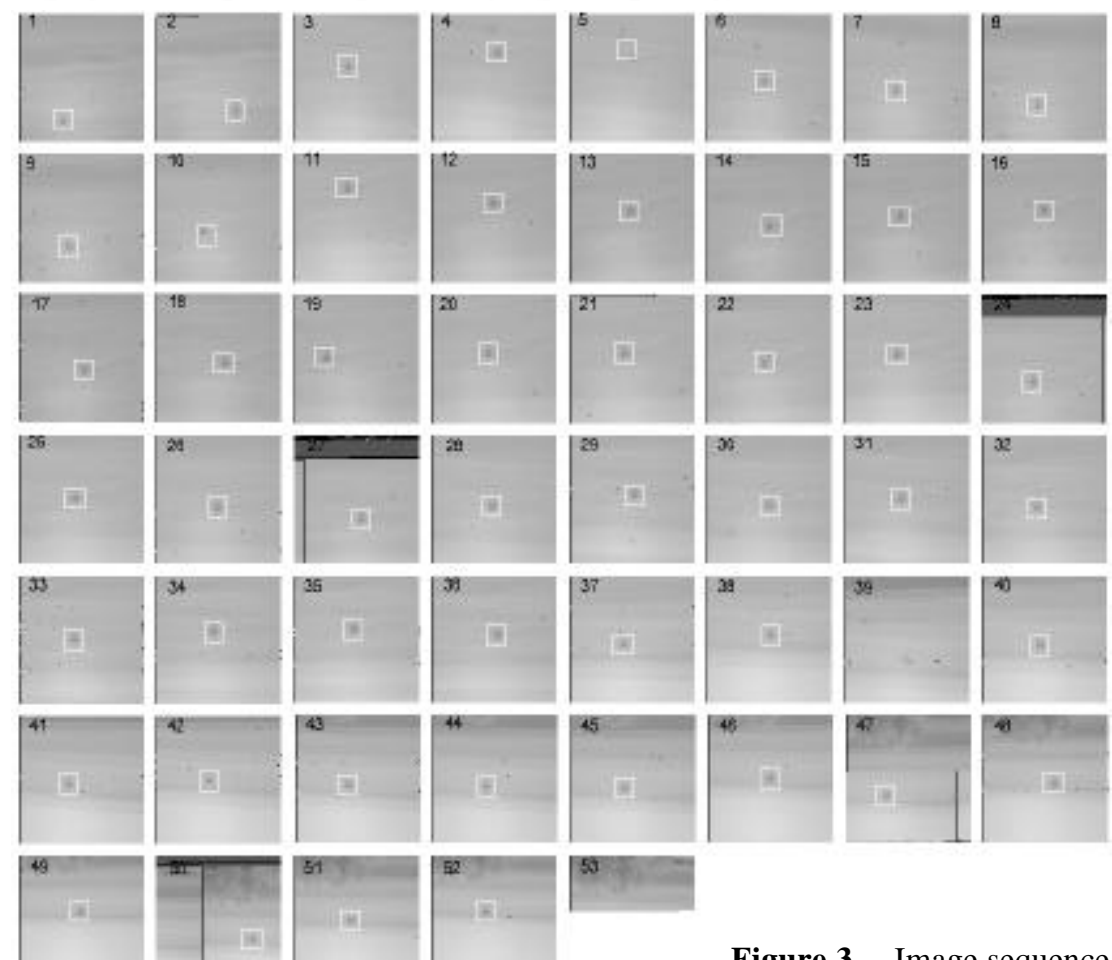
We wish to thank Mr K V S S Prasada Rao for his encouragement. We also wish to thank Mr Harish Sharma and other colleagues for their cooperation throughout this work.

\section{References}

Anuta P E 1970 Spatial registration of multispectral and multitemporal digital imagery using fast fourier transform techniques. IEEE Trans. Geosci. Electron. GE-8: 353-368

Anandan P 1985 Computing dense displacement fields with confidence measures in scenes containing occlusion. IUS workshop, DARPA, pp 236-246

Barnea D I, Silverman H F 1972 A class of algorithms for fast digital image registration. IEEE Trans. Comput. C-21: 179-186

Gonzalez R C et al 1999 Digital image processing ISE edn (Menlo Park, CA: Addison-Wesley)

Kumar S V R et al 1987a Performance studies on image registration algorithms. Proc. Int. Symp. Opto-electronic Imaging (ed.) D P Juyal (New Delhi: Tata McGraw-Hill)

Kumar S V R et al 1987b Architectures for real-time image registration. Proc. Int. Symp. Optoelectronic Imaging (ed.) D P Juyal (New Delhi: Tata McGraw-Hill)

Phillips D 1995 Image processing in C" (New Delhi: BPB)

Pratt W K 1974 Correlation techniques of image registration. IEEE Trans. Aerosp. Electron. Syst. AES-10: 353-358

Raju P V S 1990 Multiple stereo for the estimation of terrain geometry. M Tech thesis, Indian Institute of Technology, Kharagpur

Venkateswarlu R, Venkateswararao B, Raju P V S 1992 Real time hardware implementation for area correlation tracker. SPIE Vol. 1697: Acquisition, tracking and pointing VI (Washington, DC: SPIE) pp 224-234 